\title{
Modelo comunitario para la promoción, prevención, asistencia y protección ante situaciones de abuso sexual contra niñas, niños $\mathrm{y}$ adolescentes
}

I ${ }^{1}$ Gabriela Wald, 2 Ana Clara Camarotti, ${ }^{3}$ Alejandro José Capriati, ${ }^{4}$ Ana Lia Kornblit I

Resumen: Presentamos aquí un modelo teórico para la construcción de respuestas comunitarias a problemas de salud integral adolescente. El mismo condensa el trabajo realizado por nuestro equipo a lo largo de los últimos 15 ańos, tanto en el ámbito de la investigación como en la asistencia técnica a programas de intervención en Argentina. El modelo está organizado en 3 ejes conceptuales de los cuales se desprenden 10 principios y 6 pasos, los cuales recuperan los debates de la promoción de la salud, la salud colectiva y la pedagogía crítica. Si bien el mismo puede ser utilizado para trabajar distintas problemáticas de salud integral adolescente, se muestra en este artículo aplicado a un tema concreto y de relevancia internacional: el abuso sexual contra niños, niñas y adolescentes. Esta modelización supone una dialéctica que, por un lado, da lugar para que lo singular y contingente de cada experiencia pueda aparecer. No obstante, reconoce que muchos problemas se repiten en distintas comunidades, ya sea cercanas o geográficamente alejadas, y que la experiencia en el abordaje de los mismos puede aportar a construir nueva acción informada en la práctica. Proponemos aquí un trabajo basado en la inclusión de las diferencias, donde se interroguen los mitos, las creencias, los prejuicios y los estereotipos que sostienen las múltiples discriminaciones que pueden sufrir las personas.

> Palabras clave: modelo; promoción de la salud; abuso sexual infantil; adolescencia - comunidad

\author{
1 Instituto de Investigaciones \\ Gino Germani, Facultad de \\ Ciencias Sociales, Universidad \\ de Buenos Aires, Argentina \\ (gawald@gmail.com). \\ ORCID: 0000-0002-0869-1892 \\ ${ }^{2}$ Instituto de Investigaciones \\ Gino Germani, Facultad de \\ Ciencias Sociales, Universidad \\ de Buenos Aires, Argentina \\ (anaclaracamarotti@gmail.com). \\ ORCID: 0000-0003-4428-5768 \\ ${ }^{3}$ Instituto de Investigaciones \\ Gino Germani, Facultad de \\ Ciencias Sociales, Universidad \\ de Buenos Aires, Argentina \\ (alejandrocapriati@gmail.com). \\ ORCID: 0000-0001-8204-6878 \\ ${ }^{4}$ Instituto de Investigaciones \\ Gino Germani, Facultad de \\ Ciencias Sociales, Universidad \\ de Buenos Aires, Argentina \\ (alkornblit@gmail.com). \\ ORCID: 0000-0002-4647-2973
}

Recebido em: 11/07/2018 Aprovado em: 07/10/2018 Revisado em: 06/12/2018 


\section{Introducción}

En este artículo presentamos un modelo teórico ${ }^{1}$ para la construcción de respuestas comunitarias a problemas de salud adolescente y ejemplificamos el modo en que podría aplicarse para el abordaje de una temática específica: el abuso sexual contra nińas, niños y adolescentes (en adelante ASI). El mismo condensa el trabajo realizado por nuestro equipo de trabajo a lo largo de los últimos 15 ańos, tanto en el ámbito de la investigación como en la asistencia técnica a programas de intervención en distintas provincias de Argentina.

Durante este tiempo hemos podido constatar, tal como lo afirma la literatura, que los cambios en los modos de entender la salud que se han dado a partir de la segunda mitad del siglo XX, cuando la misma comenzó a entenderse como un proceso colectivo e integral influido por múltiples aspectos - y ya no sólo como ausencia de enfermedad - no han sido sencillos de traducir en las prácticas del campo (AYRES; PAIVA; FRANCA JR., 2012; KORNBLIT, 2010; POLLAND, 2007; SPINELLI, 2010). Así, a pesar de definirse la salud en términos positivos, la mayor parte de las prácticas continúan organizándose alrededor de conceptos de enfermedad (CZERESNIA, 2006) y gran parte de los programas que se autodenominan de promoción de la salud son intervenciones donde se privilegia la transmisión de información para modificar comportamientos, utilizando modelos teóricos de base conductista e individualista que la retórica en promoción de la salud hace décadas critica como poco efectivos. ${ }^{2}$ De este modo, muchos de los conceptos de la nueva salud pública - promoción de la salud, participación comunitaria, intersectorialidad, empoderamiento, etc. - se transforman en significantes "vacíos de sentido" donde los "qué", los "por qué", los "para qué", los "con quién" y los "para quién" específicos de cada situación de intervención suelen interrogarse poco o nada, configurándose una lógica de "políticas sin sujetos" (SPINELLI, 2010, p. 279).

Enmarcado en estos debates, hemos desarrollado un modelo de intervención comunitario informado tanto por la teoría en promoción de la salud y en salud colectiva - en particular por los enfoques sobre vulnerabilidad y derechos humanos (GRUSKIN y TARANTOLA, 2012) - como por nuestras experiencias de trabajo para abordar situaciones de salud integral adolescente, en su mayoría en poblaciones donde los derechos son altamente vulnerados. El mismo tiene antecedentes en trabajos teóricos previos de nuestro equipo (KORNBLIT, 2010; CAMAROTTI; 
KORNBLIT, 2015; WALD, 2015; CAPRIATI, et al., 2015), si bien se afianzó en una experiencia reciente que hemos desarrollado en tres provincias del noroeste argentino que conforman el Valle Calchaquí, convocados por Unicef Argentina entre los años 2014 y 2017. En este proyecto acompañamos el trabajo de un grupo de organizaciones sociales y gobiernos locales para la construcción de una respuesta integral frente al ASI, con un fuerte anclaje en la movilización comunitaria.

El modelo está organizado en tres ejes conceptuales de los cuales se desprenden diez principios y seis pasos. Desgrana conceptos y modos de aplicar los mismos en la práctica, a la vez que operacionaliza aquellos significantes tan hablados pero poco puestos en acción. Si bien el mismo puede ser utilizado para trabajar distintas problemáticas de salud integral adolescente, se muestra en este artículo aplicado a un tema concreto y de relevancia internacional como es el abuso sexual contra niños, niñas y adolescentes.

Se presentan en primer lugar y de manera sintética los ejes conceptuales y principios del modelo, pues enmarcan los "por qué" del tipo de intervención que proponemos. No obstante, el foco del artículo está puesto en los seis pasos del mismo, los cuales suponen avanzar en los "cómo" de la movilización comunitaria, teniendo en cuenta advertencias y consejos que hemos cosechado en nuestras experiencias de trabajo. El objetivo del modelo no es ofrecer una "receta", pues sabemos que cada comunidad es singular y que debe darse el espacio para la reflexión sobre qué quiere hacer y con quiénes. No obstante, buscamos darle lugar al hecho de que los modos en que se presentan las problemáticas sociales y de salud muchas veces son similares en distintas comunidades, ya sea que las mismas se encuentren próximas o alejadas geográficamente. Apostamos entonces a tomar en cuenta aprendizajes que puedan iluminar zonas comunes para la acción colectiva, sin descuidar lo contingente que cada territorio y cada población puedan aportar.

\section{Las ideas y teorías que inspiran este modelo: ejes conceptuales y principios}

El modelo de movilización comunitaria para el abordaje de la salud y los derechos en la adolescencia que presentamos supone comprender: a) que la salud es un proceso colectivo e integral, influido por aspectos económicos, políticos, psico-sociales, culturales y ambientales; b) que son centrales para el cuidado de la salud el apoyo social y la red de relaciones con que cuentan las personas, así como las normas de los 
grupos a los que pertenecen; c) que la comunidad es un espacio social en construcción permanente, atravesado en distintos grados por la diversidad, el conflicto y la cooperación, y no un grupo homogéneo; d) que en los vínculos comunitarios se ponen en juego intereses a veces contrapuestos y se ejercen relaciones de poder entre actores e instituciones que es preciso tener en cuenta; e) que la acción no es siempre racional y que obedece a intereses que muchas veces no pueden explicitarse; f) que no existe una sola adolescencia/juventud sino múltiples, y que la experiencia de ser joven depende de cuestiones estructurales y culturales: el lugar donde se vive, la educación a la que se accede, el trabajo de los miembros de la familia, la seguridad social con la que se cuenta, el entorno ambiental, los usos del tiempo libre, los universos de creencias, los valores a los que se adscribe, la participación en instituciones, entre otros aspectos; g) que las relaciones de género permean todos los vínculos humanos y que están constituidas histórica y socialmente, expresando relaciones de poder favorables para los varones heterosexuales y discriminatorias para las mujeres y para las personas que se alejan de la matriz heterosexual; y h) que es preciso reconocer la dignidad y la capacidad de acción de todos los actores sociales, incluyendo al mundo adolescente, mediante el fomento de su ciudadanía y participación.

Antes de adentrarnos en el modelo, queremos llamar la atención sobre los límites y alcances que este tipo de intervenciones presenta. Recuperando una de las discusiones fundamentales de las ciencias sociales, la que se pregunta en qué medida las condiciones estructurales de existencia influyen en la capacidad de acción de los sujetos, podemos decir que para que mejoren las condiciones estructurales que determinan los problemas de salud adolescente es necesario que se establezcan modificaciones sustantivas en relación con la calidad de vida de las personas, pues esta es la base de dichos problemas. No obstante, queremos recuperar la distinción que Onetto (2003) realiza entre la "zona de preocupación" y la "zona de incumbencia”. La primera comprende todos los factores que pueden influir, en este caso, sobre la salud integral adolescente, las cuales escapan a las acciones que podemos llevar a cabo con modelos de intervención de este tipo, pero que sabemos tienen una influencia decisiva sobre ella - como por ejemplo el estrato socioeconómico de las familias de los y las adolescentes. La zona de incumbencia, en cambio, es la que está directamente en el foco de lo que podemos modificar, por ejemplo, la posibilidad de que los y las adolescentes defiendan su derecho a la tener una sexualidad libre de violencia. Teniendo en cuenta la posibilidad de las intervenciones 
que pueden incentivar cambios, preferimos llamarla "zona práctica de influencia". Si bien no podemos modificar las condiciones estructurales, sí podemos generar transformaciones intermedias: reflexión crítica de la comunidad, movilización de la misma para conseguir que se amplíen derechos o que se hable de temas silenciados, trabajo con instituciones clave para mejorar los sistemas de respuestas a determinada problemática, la generación de vínculos entre adolescentes y adultos que redunden en vínculos de cuidado, entre otras.

A continuación presentamos la primera parte de nuestra modelización, la que describe los tres ejes conceptuales y diez principios que conforman el marco de ideas que movilizan la acción.

\section{Cuadro 1. Eje conceptual 1: NADA SE SOSTIENE EN EL TIEMPO SIN LA MOVILIZACIÓN COMUNITARIA, LA ARTICULACIÓN ENTRE INSTITUCIONES Y EL COMPROMISO DEL ESTADO}

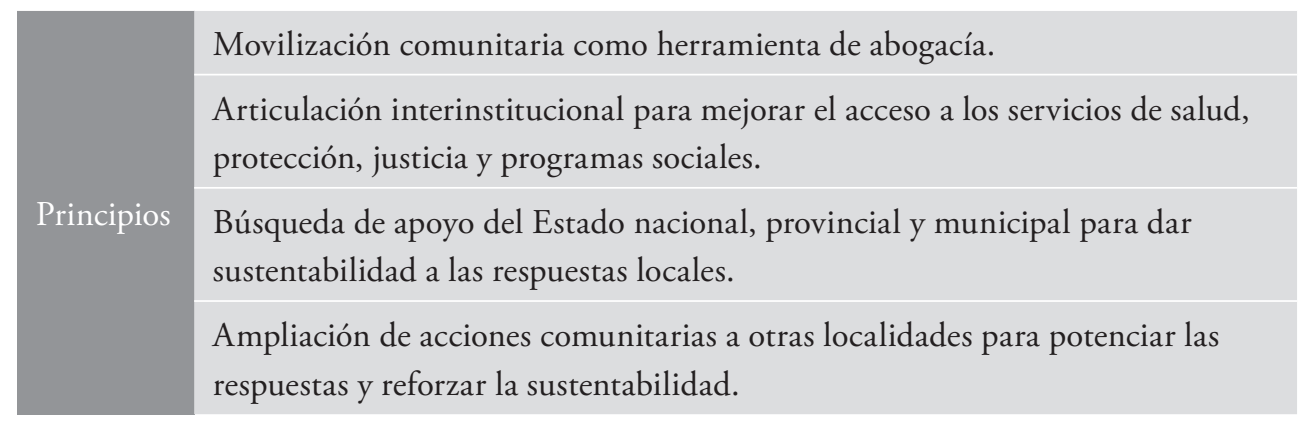

El derecho de las y los adolescentes a ejercer niveles cada vez mayores de responsabilidad y autonomía en lo que refiere a su vida y a la de sus comunidades no reduce las obligaciones de los Estados de garantizar su protección. La movilización comunitaria implica trabajar con todos los actores que tengan un compromiso con el cuidado de la salud integral de las y los adolescentes, y con la promoción de sus derechos.

Una comunidad movilizada tiene más herramientas para demandar al Estado los derechos incumplidos, así como para cuidar a sus miembros. Asimismo, cuanto más articuladas estén las instituciones de una comunidad, más protegidos estarán sus miembros. Como se explicitará al detallar las acciones en los pasos del modelo, si bien la participación comunitaria es uno de los pilares de la sustentabilidad, el otro aspecto decisivo es el apoyo del Estado. 
Cuadro 2. Ejeconceptual2:ADOLESCENTESYJÓVENESCOMOPROTAGONISTAS DEL CAMBIO, CON APOYO DE ADULTOS QUE ESTIMULAN Y CUIDAN

\begin{tabular}{ll} 
Adolescentes que tomen la palabra y sean parte de la acción colectiva. \\
\hline Principios & Escuelas como espacios privilegiados para la promoción de la salud. \\
\cline { 2 - 2 } & $\begin{array}{l}\text { Los cambios en los repertorios culturales sólo son posibles si se propicia la } \\
\text { reflexión crítica. }\end{array}$
\end{tabular}

Es sabido que los procesos de cambio en las comunidades son más duraderos si las mismas participan desde el comienzo del proceso, incluyendo la etapa diagnóstica (COLMENARES, 2011). Uno de los puntos centrales que enfatiza este modelo es la necesidad de integrar en dicha participación a jóvenes y adultos. Para que esto sea posible, hace falta lograr una valoración mutua de intereses y opiniones; sería deseable que ambos pudieran verse como otros con quienes vale la pena el intercambio.

Ahora bien, ¿cuál es el rol del mundo adulto en la movilización de los y las jóvenes? En primer lugar, es preciso facilitar una escucha atenta, comprometida y que tienda a la integración de los adolescentes en las instituciones y actividades pensadas para ellos, así como generar una progresiva autonomía que les permita crear y pensarse cada vez más como colectivo.

El mundo adulto tiene el desafío de propiciar la reflexión sobre cuál es su lugar y en qué medida el modo en que cumplen su rol habilita o no la participación genuina de los adolescentes, da lugar a su voz y fundamentalmente a sus prácticas - aún cuando las mismas les puedan generar rechazo o incomodidad. Las dudas e incertidumbres de los adultos frente al mundo cambiante en el que nos toca vivir pueden llevar por un lado, a adoptar posturas rígidas y censurantes frente a conductas adolescentes que les parecen inadmisibles, pero también pueden llevar a adoptar una postura de tolerancia extrema frente a las conductas contestatarias. En ambos casos no estaremos cumpliendo con lo que las y los adolescentes necesitan: que sostengamos de un modo firme y no rígido el lugar de las normas.

En las intervenciones comunitarias de promoción de la salud se busca propiciar cambios en las prácticas sociales, lo que en gran medida también transforma sus repertorios culturales. Es preciso reflexionar sobre las raíces culturales, interaccionales y sociales que enmarcan las prácticas que queremos modificar. Como veremos más adelante, limitar este proceso a lo que se ha denominado "modelo informativo" no ha 
dado buenos resultados. Es importante alentar las capacidades de las comunidades mediante la reflexión crítica, identificar códigos y escenas institucionales que no son propicias para abordar el problema que se quiere trabajar, así como identificar repertorios positivos para la promoción y el cuidado de las comunidades frente a la problemática seleccionada.

\section{Cuadro 3. Eje conceptual 3: DIAGNOSTICAR, PLANIFICAR, EVALUAR y SISTEMATIZAR DE MANERA PARTICIPATIVA}

Conformación y capacitación de equipos locales para llevar adelante las tareas
y unificar criterios de intervención.

La base de toda acción de movilización comunitaria es la participación de la misma en todas las fases del proceso. Entendemos la participación como un proceso en el cual se involucra a los actores de una comunidad no sólo para conocer su opinión sino también para que formen parte de los procesos de toma de decisiones. Así como es importante diagnosticar y planificar con los aportes de los distintos actores de una comunidad, lo es que puedan ser parte de los procesos de monitoreo, evaluación y sistematización (NIREMBERG, 2009; KORNBLIT et al., 2016).

La sistematización de experiencias implica reconstruir información sobre los actores y factores que han intervenido en un proyecto, sobre cómo se han vinculado entre sí y, en la medida de lo posible, analizar por qué motivos lo han hecho de esa manera. La clave es avanzar en una interpretación crítica del proceso que permita comprender, interpretar y extraer aprendizajes que tengan utilidad para el futuro.

\section{El paso a paso del modelo}

Presentamos aquí una serie de pasos que permiten implementar los principios arriba sintetizados. Los mismos no son una unidad sucesiva que debe seguirse sino una secuencia lógica para organizar la acción. Los primeros tres pasos respetan una secuencia temporal, mientras que los pasos 4,5 y 6 coexisten y se hacen en simultáneo, constituyendo el núcleo central de la intervención comunitaria. 
El siguiente esquema muestra el modo en que se vinculan los pasos del modelo, enmarcados en los ejes y principios detallados en el apartado anterior:

\section{Figura 1. Esquema de los pasos del modelo de acción comunitaria para el trabajo sobre abuso sexual infanto juvenil}

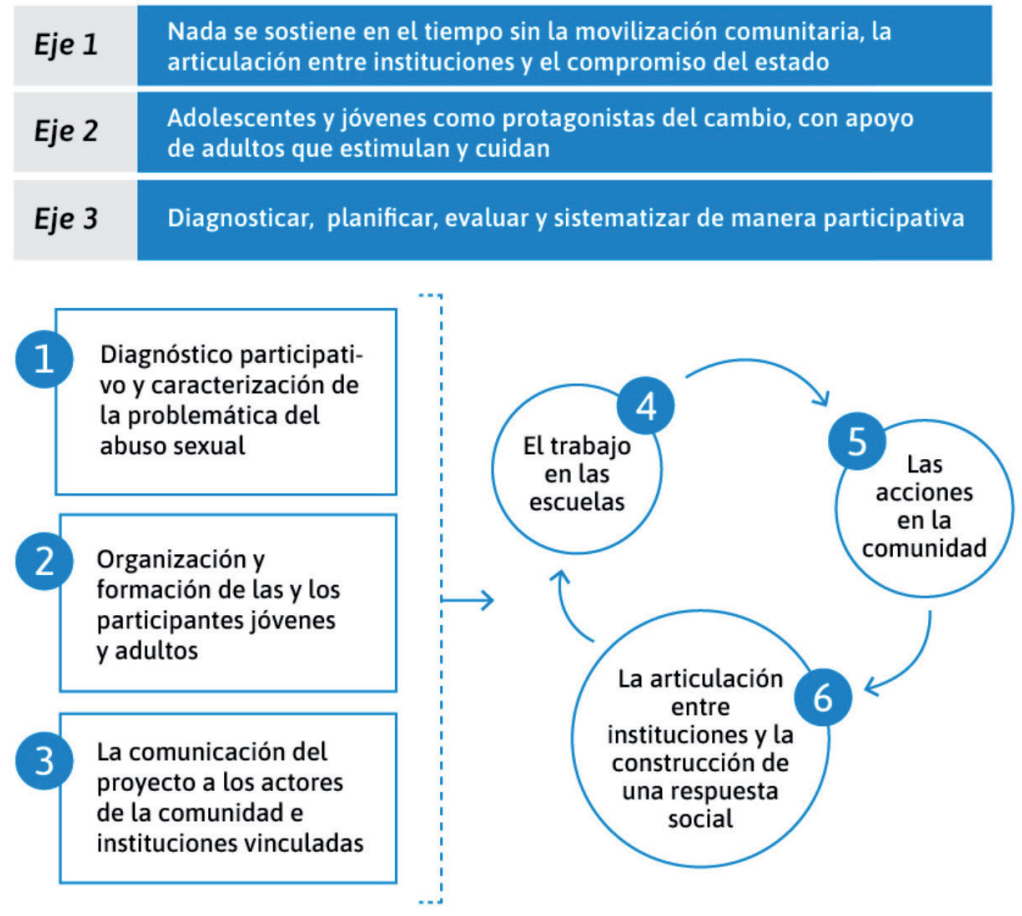

Los ejes y principios del modelo argumentan los "por qué" y "para qué" de las acciones de promoción, prevención y cuidado, es decir, las bases conceptuales de la acción. Los pasos, por su parte, implican avanzar sobre los "cómo", pensando en sujetos, comunidades y problemáticas concretas. Estos pasos son lo suficientemente generales como para dar lugar a quienes lleven adelante un proyecto a definir las actividades específicas al interior de cada uno, o incluso proponer nuevos en función del escenario que afronten, de los recursos de los que dispongan, y de los grupos, equipos e instituciones que estén dando forma a la nueva experiencia.

Los pasos, al implicar una operacionalización de la teoría en la práctica, especifican objetivos, a la vez que indicadores para la evaluación. Los mismos no agotan la 
variedad y cantidad de indicadores que cada comunidad pueda definir, no obstante, apuntan a ejemplificar los modos en que las acciones pueden ir evaluándose en cantidad y calidad, tanto en el corto como en el mediano o largo plazo.

\section{PASO 1: Diagnóstico participativo y caracterización de la problemática del abuso sexual contra niños, niñas y adolescentes}

Las intervenciones comunitarias no parten de un grado cero de organización, sino que, partiendo de aquello que existe, identifican de modo colectivo aquello que puede mejorarse, que debe cambiarse, y aquello que hay que crear para optimizar la respuesta social a una problemática dada - en el caso que nos ocupa al ASI.

El diagnóstico participativo implica el acercamiento entre organizaciones, el Estado y otros grupos de una comunidad para priorizar los principales problemas vinculados a cuestiones de salud y derechos en la adolescencia, pensar modos de encararlos y coordinar la acción de manera conjunta. En este caso, el diagnóstico implica la caracterización del ASI en la comunidad (tipo de abusos de los que se han registrado conocimiento, magnitud del problema en la comunidad, modos en los que ocurre, tratamiento de los mismos en las instituciones, judicialización de los casos, espacios privilegiados para su develamiento, etc.) y cómo se vincula con otras problemáticas (embarazo en la adolescencia precoz, violencia doméstica, suicidio, etc.). El punto de partida para un diagnóstico participativo son los conocimientos y saberes de las personas que residen en un territorio, incluyendo tanto a jóvenes como a todos aquellos que trabajen con ellos.

En este proceso es importante reunir la información disponible y la evidencia científica con los conocimientos teóricos, empíricos y vivenciales de todos los involucrados en el tema. A partir del diagnóstico se priorizarán los aspectos del tema a tratar y se hará una selección para comenzar a abordar algunos de ellos.

El diagnóstico y la priorización presentan dos grandes desafíos. Por un lado, que las instituciones y actores relevantes de una comunidad se sientan convocados por la iniciativa y aseguren su participación, aún cuando las orientaciones políticas o partidarias de las mismas sean contrastantes. Esto implica convocar y comprometer a todo aquel que trabaje cuestiones vinculadas al tema - en el caso del ASI hablamos de escuelas, servicios de protección y de salud, pero también de la justicia y la policía, actores muchas veces reacios al trabajo en red. 
En segundo lugar, hace falta contar con la presencia y la participación de adolescentes y jóvenes de la comunidad, lo cual constituye un desafío doble: que se acerquen y que hagan oír su voz. Para ello es preciso, por un lado, generar una convocatoria amplia en distintos ámbitos donde los y las adolescentes circulan (la escuela, el club, el centro cultural, el comedor, organizaciones comunitarias, etc.) y por el otro, que cuando los adolescentes se acerquen, el mundo adulto pueda ofrecerles espacios en donde se combine la reflexión con la distensión, donde vayan adquiriendo confianza progresiva para hablar de los temas que los inquietan, y donde esté cuidada la confidencialidad de aquello que decidan compartir.

Existe un conjunto amplio de técnicas que se pueden utilizar para orientar los diagnósticos participativos, desde las más conocidas de la planificación en salud y el trabajo comunitario (mapa de actores, cartografías, árbol de problemas, espina de pez, análisis FODA) hasta técnicas propias del sociodrama y la educación popular.

Recordemos que las técnicas permiten procesar datos cuantitativos y cualitativos y resultan de utilidad para informar el proceso de toma de decisiones: agrupan información dispersa, facilitan el intercambio y documentan los acuerdos y disensos. No obstante, ninguna técnica provee por sí misma los resultados que se esperan de un proceso como el que aquí se propone.

Un insumo que facilita este proceso es la elaboración de informes ejecutivos sobre las problemáticas consideradas relevantes. Los mismos proveen: definición conceptual, marco legal, datos sobre magnitudes y tendencias del problema, recursos públicos existentes y buenas prácticas para su abordaje. De este modo, colaboran en el proceso de toma de decisión y ponen a disposición la evidencia científica existente.

\section{Objetivos del diagnóstico participativo}

- Priorizar los aspectos del tema - en este caso relativos al ASI - en los que las personas convocadas estén de acuerdo en trabajar.

- Realizar debates sobre dichos aspectos, aportando información y datos relevantes.

- Formular objetivos para la acción colectiva y especificar quiénes serían las instituciones y grupos participantes.

- Constituir un grupo de trabajo con un coordinador ejecutivo (puede ser rotativo)

- Sentar las principales líneas de acción de una propuesta de trabajo colectiva 


\section{Indicadores de evaluación}

- Amplitud de la cobertura de la convocatoria realizada a actores de la comunidad involucrados en el trabajo en torno al ASI.

- Participación efectiva de diferentes sectores de la comunidad en los intercambios realizados en las etapas de diagnóstico, caracterización de problemas y priorización de los aspectos a trabajar.

- Tipo y modalidad de participación adolescente y juvenil alcanzada.

- Constitución de un grupo coordinador de las tareas.

\section{PASO 2: Organización y formación de las y los participantes jóvenes y adultos}

Luego de la etapa diagnóstica es importante realizar encuentros de formación entre equipos técnicos y no técnicos, grupos de jóvenes, trabajadores del Estado, referentes comunitarios, investigadores, docentes y profesionales para acordar una mirada, establecer funciones y responsabilidades, y generar un plan de acción colectivo. En estos encuentros se brindan insumos para definir la problemática a trabajar, así como recursos y herramientas para diseñar e implementar acciones a nivel local.

Es importante contar con una mirada compartida que trascienda las diferencias de las organizaciones que componen una comunidad. Esto no es sencillo de lograr pues como antes advertimos, en los vínculos comunitarios muchas veces aparecen intereses contrapuestos, así como racionalidades y modos de hacer heterogéneos, los cuales implican la existencia de conflictos atravesados por relaciones de poder entre actores e instituciones que coexisten en un territorio. No obstante ello, se propone aquí potenciar vínculos positivos entre los grupos sociales con los que se trabaja. Se busca maximizar las potencialidades del trabajo colaborativo a la vez que minimizar la influencia de los intereses particulares.

En los encuentros de formación y organización se puede trabajar con adolescentes y adultos en sesiones compartidas o por separado. El desafío de la tarea conjunta es doble: 1) que las y los adolescentes no reproduzcan en sus opiniones las posiciones del mundo adulto, 2) que el grupo adulto no censure la expresión de los grupos de jóvenes. En cualquiera de los dos casos, es importante, tal como lo afirma Debora Kantor (2008), generar espacios que tengan sentido para los propios jóvenes, propuestas que dialoguen con sus proyectos y necesidades, mostrando una apertura 
de la capacidad de escucha. Si las y los adolescentes tienen el derecho a participar, el mundo adulto tiene como obligación generar esos encuentros y sostenerlos, aún cuando la demanda sea baja.

Existen múltiples técnicas para facilitar la formación y la producción de conocimiento: dinámicas para vivenciar situaciones específicas, análisis críticos utilizando recursos audiovisuales, reflexión grupal para la presentación de casos, entre otras. Dentro de los encuentros es positivo incorporar algún momento de evaluación o devolución para ajustar las metodologías y los contenidos.

\section{Objetivos de la etapa de formación y organización de los equipos}

- Acordar una mirada compartida: desarrollar un marco conceptual general sobre prevención, promoción, atención y cuidados de la salud entre adultos y adolescentes.

- Desarrollar un marco conceptual específico sobre el ASI.

- Incorporar un abanico de estrategias posibles para el trabajo en la comunidad sobre el tema del ASI.

- Construir un rumbo de acción: definir estrategias y actividades, ubicándolas en un cronograma tentativo de acción.

- Establecer papeles, funciones y responsabilidades de grupos que se formen para desarrollar actividades específicas de trabajo en la comunidad.

\section{Indicadores de evaluación}

- Tipo de articulación entre los referentes adultos y los y las adolescentes que participan del proyecto.

- Modo en que se involucran los y las adolescentes y jóvenes en los inicios del proyecto, constitución o no de un grupo, con liderazgo adulto o juvenil.

- Ampliación conceptual en relación con la promoción de la salud integral adolescente y con la/s temática/s seleccionadas para trabajar, especialmente por parte del grupo coordinador del proyecto.

- Incorporación de nuevas modalidades de intervención en prevención, promoción, asistencia y protección en relación con el ASI por parte de los y las participantes adolescentes y adultos. 
- Acuerdos logrados en cuanto a las acciones a realizar y las responsabilidades a asumir por parte de los y las adolescentes, así como de las organizaciones e instituciones que participan.

- Tipo de materiales redactados por el equipo que asumirá la coordinación del proyecto: cronograma tentativo, gráficos sobre las etapas y dispositivos del proyecto, guías operativas de trabajo, son algunos ejemplos.

\section{PASO 3: La comunicación del proyecto a los actores de la comunidad e instituciones vinculadas}

La comunicación del proyecto implica dos grandes aspectos: por un lado, la instalación del tema en la comunidad para problematizarlo y comenzar a desnaturalizarlo, y por el otro, la sensibilización para lograr el compromiso por parte de las organizaciones de la comunidad e instituciones estatales en sus distintos niveles (municipales, provinciales, nacionales). Esto supone acciones que van desde la presentación del proyecto y sus objetivos ante distintos actores (el gobierno local, los servicios de salud, las escuelas, la comunidad mediante un evento público) hasta la convocatoria para participar de las actividades que se realicen en el marco del mismo, así como también los mensajes de promoción y prevención que se diseñen.

La instalación de un tema como el ASI en la comunidad es un paso central tanto para la prevención de nuevos casos como para mejorar los sistemas de respuestas, así como para demandarle al Estado que haga cumplir los derechos de las y los adolescentes. El develamiento de aquello de lo que no se habla, llevado a cabo de forma colectiva, es un poderoso instrumento en los procesos de cambio.

\section{Objetivos de la comunicación del proyecto}

- Presentar el proyecto a las organizaciones e instituciones comunitarias para lograr su compromiso con el tema.

- Difundir el proyecto y sus objetivos en la comunidad a través de medios de comunicación locales y de redes sociales para desnaturalizar el ASI y sensibilizar a la comunidad.

- Diseñar estrategias de comunicación dirigidas a diferentes grupos de la comunidad, para desnaturalizar el abuso sexual contra niños, niñas y adolescentes y para comprometer a la mayor cantidad de familias con su erradicación. 
- Diseñar mensajes de prevención del ASI y promoción de derechos, de modo consensuado entre adultos y adolescentes.

- Distribuir en la comunidad material impreso para que las instituciones y los comercios las exhiban en sus espacios, de modo tal de comprometerlas en la lucha contra el silenciamiento y la naturalización del ASI.

\section{Indicadores de evaluación}

- Actores institucionales clave incorporados al proyecto a partir de la convocatoria realizada.

- Tipo y cantidad de entrevistas de difusión realizadas a través de los medios masivos locales.

- Tipo y cantidad de mensajes preventivos diseñados y distribuidos en la comunidad.

- Logo y lema diseńados y reproducidos en los materiales del proyecto.

- Cantidad de seguidores en redes sociales.

- Cantidad de instituciones que exhibieron material de difusión del proyecto.

- Cantidad de locales comerciales que exhibieron material de difusión del proyecto.

- Modos en que la propia comunidad se ha percibido sensibilizada frente al ASI.

\section{PASO 4: El trabajo en las escuelas}

Dado que la escuela es una institución fundamental en la formación de niñas, niños y adolescentes, el trabajo en la promoción y el cuidado de la salud desde el modelo comunitario que proponemos concibe a la escuela como uno de sus espacios clave para la acción.

Existe una modalidad de trabajo que aunque ha sido duramente criticada (MORENO; GARCÍA; CAMPOS, 1994; KORNBLIT; DIZ, 2004; PAIVA, 2006) continúa aún muy extendida en la práctica: el privilegio de estrategias para ampliar la información sobre un tema o problema de salud con el objeto de impedir acciones consideradas "de riesgo", en particular entre los y las adolescentes. Muchas veces estas actividades son coordinadas por algún "experto” que cuenta cuáles son las prácticas "correctas" frente a otras consideradas desviadas o disruptivas, lo cual colabora en la construcción de estereotipos, en particular en la adolescencia y juventud. Todo esto ocurre aún cuando es sabido que la información por sí misma no modifica las prácticas, y que las técnicas que implican de modo activo a las 
personas en los procesos de aprendizaje son las que proporcionan conocimientos más sólidos y duraderos (KORNBLIT, 2017).

Para que la promoción de la salud adolescente logre los objetivos que se propone, es importante trabajar no sólo desde el modelo informativo, presentando datos e información sobre posibles daños a la salud de determinadas prácticas, sino con el modelo de empoderamiento. Este último alienta la capacidad de las personas para actuar sobre las circunstancias que afectan su vida y la de sus comunidades (DI LEO, 2009). Asimismo, considera que la modificación de las conductas no se logra mediante la persuasión sino que requiere de la participación de los sujetos en los procesos de cambio - cambios que pueden alcanzarse haciendo uso de la reflexión crítica sobre la propia vida y la de nuestros grupos de pertenencia en diálogo con otros que a los y las adolescentes les resulten significativos, ya sean pares o adultos con quienes lograron establecer relaciones de confianza.

Desde esta perspectiva, la promoción de la salud en la escuela permite el pasaje de los y las adolescentes de la categoría de objetos evaluados a la de sujetos con capacidades y potencial de acción que puede - y debe - ser puesto en movimiento. Brinda la posibilidad de revertir el binomio educador/educando tradicional por una relación dialógica, entendiendo que el saber se encuentra compartido entre docentes y alumnos y, por ende, ambos son indispensables para construir las prácticas preventivas y de cuidado.

La construcción de espacios integradores también implica una convocatoria abierta para quienes deseen participar del grupo de adolescentes que van a trabajar en y por la comunidad. Es importante que dicha convocatoria no se realice teniendo en cuenta la figura del "alumno modelo", el criterio de la buena conducta o el del rendimiento académico. Sería deseable propiciar espacios en los cuales puedan integrarse aquellos que habitualmente son señalados, identificados como conflictivos o problemáticos y dar lugar a quienes deseen ser parte de la movilización, valorando y respetando a cada uno en sus capacidades, posibilidades y diferencias, aún cuando estas puedan generar rechazo o incomodidad, sobre todo para el mundo adulto.

Las acciones que pueden llevarse a cabo en las escuelas son amplias, incluyen la formación de docentes y estudiantes en la problemática seleccionada (en este caso ASI) desde una perspectiva que fomente la reflexión crítica; la realización de proyectos educativos vinculados a dicho tema; la participación en concursos escolares; la realización de eventos, talleres, stands, recitales. Todas estas actividades tendrán una 
doble finalidad: por un lado, harán que la escuela "se abra" a la comunidad, muestre lo que está haciendo, interactúe con el territorio en el cual se localiza. Por el otro, cada acción colaborará con la sensibilización e instalación del tema en la comunidad.

Una figura clave que puede potenciar el modelo de trabajo que proponemos es el docente referente en salud, quien se encargará de propiciar la articulación entre los servicios de salud y la escuela. Esta persona es un o una docente capacitado/a en las temáticas que se trabajen, que tenga empatía con los y las adolescentes y pueda brindar una escucha activa y confidencial, a la vez que orientación e información en cuestiones vinculadas a la salud. Asimismo, se reunirá a los y las adolescentes que estén interesados en conformar un equipo de trabajo en prevención y promoción dirigido a sus pares. Finalmente, la convocatoria a padres y madres de la comunidad interesados/ as es otro elemento que potencia la movilización de las instituciones escolares.

Los modos en que el equipo coordinador del proyecto articula con las autoridades escolares para definir roles y responsabilidades dependerán de cada comunidad, de los vínculos previos entre instituciones, de las fortalezas de cada uno de los actores y de aspectos que aporta la contingencia del encuentro entre actores e instituciones en su singularidad.

\section{Objetivos del trabajo en la escuela}

- Fortalecer el vínculo entre escuela, familia y comunidad, y las metodologías activas de enseńanza-aprendizaje en torno a aspectos de la salud priorizados por consenso.

- Generar vínculos positivos entre docentes y alumnos que contribuyen a la promoción de climas sociales escolares favorables, en los cuales se priorice la escucha y el diálogo.

- Lograr la vinculación entre los sectores educación y salud, para lo cual es clave la figura del docente referente en salud.

\section{Indicadores de evaluación}

- Cantidad de escuelas en las que se trabajó.

- Cantidad y tipo de talleres de formación realizados con docentes y con estudiantes.

- Cantidad de docentes capacitados en la temática del ASI.

- Cantidad de docentes referentes de salud en las escuelas. 
- Cantidad de adolescentes formados como multiplicadores de pares en salud, en particular en prevención del ASI.

- Grado de autonomía que se ha logrado por parte de los adolescentes en relación con sus actividades, siempre integradas en el proyecto general de trabajo comunitario.

- Alcances de la articulación escuela-equipos de salud lograda.

\section{PASO 5: Las acciones en la comunidad}

Las acciones que se realizan en la escuela se complementan con aquellas que puedan llevarse a cabo en ámbitos comunitarios, las cuales en conjunto contribuirán a sensibilizar a la población y desnaturalizar situaciones que generan sufrimiento y que se hallan silenciadas, como es el caso del ASI. Mediante estas acciones, se trata de proveer a las poblaciones de herramientas para la solución de problemas, para la toma de decisiones, y de metodologías participativas como medio para fortalecerlas.

Dentro de las acciones de movilización comunitaria se encuentran eventos masivos en los pueblos, barrios o ciudades donde tiene lugar un proyecto; talleres que se den en organizaciones de la sociedad civil; campamentos u otros encuentros de jóvenes promovidos por los proyectos; realización de actividades culturales o recreativas para adolescentes donde además de una disciplina o un deporte se trabaje el tema en el cual se quiere sensibilizar, bicicleteadas, ferias u otras actividades de visibilización que impliquen a distintos actores trabajando en conjunto, entre muchas que cada comunidad podrá imaginar.

Existe un abanico de actividades que podrán ser organizadas en conjunto entre adolescentes y adultos y otras en las cuales se espera que los primeros, de manera progresiva, vayan liderando y planificando de manera cada vez más autónoma. Pues para los y las adolescentes la posibilidad de concretar acciones colectivas en las cuales se visibiliza su trabajo impacta de manera positiva en sus posiciones en el mundo. Les muestra que son capaces y que tienen posibilidades de construir de manera colectiva.

Al diseñar cualquier actividad deben tomarse en cuenta herramientas de la planificación acción participativa (COLMENARES, 2011) para definir qué se quiere hacer, cuál es el objetivo, a quiénes va dirigida, cómo se va a convocar a los participantes, qué dinámicas de trabajo se van a utilizar, dónde y cuándo se realizará, qué recursos económicos se necesitarán, así como los modos en que se va a registrar o evaluar la acción. 


\section{Objetivos de las acciones en la comunidad}

- Articular todas las actividades que se realicen en relación con la temática en un proyecto mayor que se difunda en la comunidad.

- Priorizar el proyecto en la agenda local.

- Que los adultos acompañen las actividades programadas de los jóvenes en las primeras etapas del proyecto.

- Lograr la autonomía progresiva de los jóvenes en sus actividades proyectadas, siempre con el acompańamiento de los adultos.

\section{Indicadores de evaluación}

- Cantidad de instituciones involucradas en las actividades comunitarias.

- Cantidad y calidad de las actividades organizadas por adolescentes.

- Cantidad y calidad de las actividades realizadas en conjunto entre adultos y adolescentes.

- Grado de autonomía de los y las adolescentes en la programación y realización de actividades programadas.

- Grado de acompańamiento adulto en las actividades programadas y realizadas por los jóvenes.

\section{PASO 6: La articulación entre instituciones y la construcción de una respuesta social frente al ASI}

La articulación es una herramienta fundamental para la acción territorial, y sólo cuando la misma se logra es posible brindar respuestas integrales. En este modelo, la articulación trasciende la apelación tradicional a la intersectorialidad y enfatiza la necesidad de lograr la articulación en distintos niveles:

- entre instituciones estatales como escuela, servicio de salud, protección o desarrollo social;

- entre organizaciones de la sociedad civil e instituciones estatales;

- entre actividades como promoción, prevención, asistencia y protección;

- entre adolescentes y adultos;

- entre disciplinas.

En la gestación de cualquier trabajo comunitario está la voluntad de aunar los esfuerzos y las acciones de los distintos grupos e instituciones. No obstante ello, y 
los beneficios reconocidos de la articulación en todos sus niveles, las dificultades que aún conlleva ponerla en práctica son notables. Comprender que la acción de cada equipo, servicio o institución forma parte de un engranaje mayor es uno de los principales desafíos.

Es importante enfatizar la centralidad que para este modelo tiene el encuentro entre el Estado y las organizaciones comunitarias, pues mientras estas últimas logran una cercanía territorial, el Estado puede garantizar la continuidad y sostenibilidad de las respuestas elaboradas localmente. La articulación es indispensable en casos de ASI, donde, por ejemplo, alguna dificultad o traba en el sistema judicial o en la radicación de la denuncia puede minar la confianza de la comunidad en las instituciones y personas que están trabajando para que la respuesta social sea más adecuada. La articulación interinstitucional, intersectorial y el diálogo con las autoridades judiciales provinciales pueden resultar decisivos para que las acciones emprendidas no caigan en saco roto.

\section{Objetivos de la articulación}

1. Vincular instituciones para facilitar el acceso de la población a servicios de salud y protección.

2. Garantizar espacios de reunión para planificar actividades, gestionar acuerdos, organizar convocatorias, responder a demandas y casos críticos, informar sobre lo realizado, etc.).

3. Elaborar una respuesta social consensuada en relación con la problemática trabajada, en este caso en relación con el ASI.

4. Regionalizar las acciones y establecer acuerdos provinciales para potenciar las respuestas y reforzar la sustentabilidad de las acciones locales.

\section{Indicadores de evaluación}

- Convenios explícitos de articulación entre instituciones de la comunidad logrados.

- Tipo y cantidad de acuerdos con instituciones previamente no involucradas en respuestas consensuadas frente a casos de ASI.

- Protocolos interinstitucionales de respuesta frente a casos de ASI diseñados, difundidos y puestos en práctica.

- Cantidad y tipo de articulación con instituciones provinciales y/o del gobierno nacional. 


\section{Teorizar la práctica y practicar la teoría: a modo de cierre}

Este trabajo sintetiza un modo de hacer prácticas preventivas, de promoción y protección en temas de salud adolescente, ejemplificando su uso para la prevención del ASI. La potencia de esta modelización está dada por el modo en el que fue construida, pues es producto del intercambio con actores de comunidades diversas con quienes compartimos experiencias de trabajo comunitario, así como de reflexiones sobre la práctica que hemos iluminado con aportes teóricos de las ciencias sociales aplicadas a la salud. Esto último resulta fundamental pues entendemos que la salud se encuentra estrechamente vinculada con las condiciones en las que se desarrolla la vida de las personas, con el apoyo social y la red de relaciones con las que cuentan las mismas, y con las normas de los grupos a los que pertenecen. Por eso, y tal como lo postulan AYRES, PAIVA y FRANCA JR (2012) las acciones que se lleven a cabo en salud no pueden pensarse por fuera de la interacción entre los aspectos subjetivos, programáticos y sociales de los grupos con los que se trabaja. Pues es preciso comprender tanto las bases sociales como las implicancias éticas y políticas que subyacen a las prácticas de cuidado de las personas y comunidades.

Como se observó a lo largo del artículo, el modelo que presentamos consta de dos partes. Una primera, expuesta de manera muy sintética, en la cual a partir del diálogo con la teoría en ciencias sociales y salud hemos construido los tres ejes conceptuales y los diez principios que guían el modelo. Y una segunda parte, en la cual este trabajo hace foco, donde ofrecemos una secuencia de pasos posibles para promover salud y cuidados de manera colectiva, y donde identificamos objetivos e indicadores para avanzar en la implementación de un sinnúmero de ideas sobre las cuales se ha escrito mucho y hecho no tanto. La idea es ofrecer lineamientos para convertir la teoría en prácticas concretas, y para reforzar la teoría a partir de las diversas prácticas en las que hemos estado involucrados como equipo.

Los seis pasos expuestos en este artículo suponen una dialéctica que, por un lado, da lugar para que lo singular y contingente de cada experiencia pueda aparecer. No obstante, reconocen también que muchos problemas se repiten de modo similar en distintas comunidades, ya sea cercanas o geográficamente alejadas, y que la experiencia en el abordaje de los mismos puede aportar a construir acción informada en la práctica. La propuesta no apunta a encorsetar soluciones, pero sí a tomar en cuenta aprendizajes previos que puedan iluminar zonas o espacios comunes de acción. 
Muchas veces en las prácticas de promoción de la salud subyace un modelo basado en estereotipos donde existe una única manera de comportarse que es "correcta". Así, muchos piensan que hay un "deber ser" que transmitir a través de información, y todo lo que no entre en ese "deber ser" es visto como disruptivo o desviado. Frente a esto proponemos un modelo de trabajo basado en la inclusión de las diferencias, donde quien quiera participar pueda hacerlo, y donde quienes participen encuentren un lugar de escucha sin prejuicios. Este modelo implica tratar de derribar los mitos, creencias, prejuicios y estereotipos que sostienen las múltiples discriminaciones que pueden sufrir las personas. Finalmente, al ser un modelo alineado con el enfoque de Derechos Humanos, exhibe la responsabilidad de los estados y gobiernos en los procesos de salud-enfermedad-cuidado. ${ }^{3}$

\section{Agradecimientos}

El proceso de intervención que permitió consolidar esta modelización fue financiado por UNICEF Argentina. Nuestro agradecimiento a quienes fueron los protagonistas en este proceso comunitario regional, los integrantes de la "Red Aquí y Ahora a Tu Lado” de Santa María (Catamarca); la comunidad indígena de Amaicha del Valle (Tucumán) y los gobiernos municipales de San Carlos y Cafayate (Salta).

\section{Referencias}

AYRES, J. R.; PAIVA, V.; FRANCA JR, I. Conceitos e práticas de prevençấo: da história natural da doença ao quadro da vulnerabilidade e direitos humanos. In: PAIVA, V.; AYRES, J. R.; BUCHALLA, C. (Orgs.). Vulnerabilidade e direitos humanos. Prevenção e promoção de saúde, Livro 1. Curitiba: Juruá Editora, 2012. p. 71-94.

CAMAROTTI, A. C.; KORNBLIT, A. L. Abordaje integral comunitario de los consumos problemáticos de drogas: construyendo un modelo. Salud Colectiva, Buenos Aires, v. 11, n. 2, p. 211-221, 2015. Disponível em: <http://revistas.unla.edu.ar/saludcolectiva/article/view/684>. Acesso em: 27 fev. 2018.

CAPRIATI, A. et al. La prevención de los consumos problemáticos de drogas desde una perspectiva comunitaria: un modelo para armar. Revista Argentina de Salud Pública, v.6, n. 22, p. 21-28, mar 2015. Disponível em: <http://www.rasp.msal.gov.ar/rasp/articulos/ volumen22/21-28.pdf>. Acesso em: 27 fev. 2018.

COLMENARES, A. Investigación-acción participativa: una metodología integradora del conocimiento y la acción. Voces y Silencios: Revista Latinoamericana de Educación, v. 3, n. 1, p. 102-115, 2011. 
CZERESNIA, D. El concepto de salud y la diferencia entre prevención y promoción. In: CZERESNiA, D.; FREITAS, M. (Orgs.). Promoción de la salud. Conceptos, reflexiones, tendencias. Buenos Aires: Lugar Editorial, 2006. p. 47-64.

DI LEO, P. La promoción de la salud como política de subjetividad: constitución, límites y potencialidades de su institucionalización en las escuelas. Salud Colectiva. Buenos Aires, v. 5, n. 3, 2009. Disponível em: <http://www.scielo.org.ar/scielo.php?script=sci_arttext\&pid =S1851-82652009000300006>. Acesso em: 27 feb. 2018.

DI VIRGILIO, M.; SOLANO, R. Monitoreo y evaluación de politicas, programas y proyectos sociales. Buenos Aires: Fundación CIPPEC, 2012. Disponível em: <https://www.unicef.org/ argentina/spanish/cippec_uni_monitoreo_evaluacion.pdf>. Acesso em: 27 feb. 2018.

GRUSKIN, S.; TARANTOLA, D. Un panorama sobre saúde e directos humanos. In: PAIVA, V.; AYRES, J. R.; BUCHALLA, C. (Orgs.). Vulnerabilidade e direitos humanos. Prevenção e promoção de saúde, Livro 1. Curitiba: Juruá Editora, 2012. p. 23-42.

KANTOR, D. Variaciones para educar adolescentes y jóvenes. Buenos Aires: Del Estante Editorial, 2008.

KORNBLIT, A. L. Escuelas comprometidas en prevención, promoción y cuidado de la salud. Acta Psiquiátrica y Psicológica de América Latina, v. 63, n. 3, p. 203-211, 2017.

. La promoción de la salud entre los jóvenes. Acta Psiquiátrica y Psicológica de América Latina, v. 56, n. 3, p. 217-225, 2010.

KORNBLIT, A. L. et al. Una propuesta para sistematizar experiencias en el abordaje comunitario de los consumos problemáticos de drogas. Buenos Aires: Teseo, 2016. Disponível em: <https:// www.editorialteseo.com/archivos/14385/abordaje-comunitario-de-los-consumos-de-drogas/>. Acesso em: 27 feb. 2018.

KORNBLIT, A. L.; DIZ, A. M. Teoría y práctica en promoción debla salud: el caso del consumo abusivo de drogas. In: KORNBLIT, A. L. (Coord.). Nuevos estudios sobre drogadicción. Buenos Aires: Biblos, 2004. p.111-120.

MORENO, A. S.; GARCÍA, E. R.; CAMPOS, P. M. La actitud participativa en salud: entre la teoría y la práctica. Murcia: Universidad de Murcia, 1994. 176 p.

NIRENBERG, O. Evaluación y participación: orientaciones conceptuales para una mejora de la gestión. In: CHIARA, M.; DI VIRGILIO, M.; ARRIAGADA, I. Gestión de la politica social: conceptos y herramientas. Buenos Aires: Prometeo Libros, 2009. p. 416.

ONETTO, Fernando. Criterios de intervención en las problemáticas de convivencia escolar. In: Monografías virtuales. Ciudadanía, democracia y valores en sociedades plurales, Organización de Estados Ibero Americanos (OEI), Area de Cooperación Educativa, 2003. Disponível em: http:// www.oei.es/historico/valores2/monografias/monografia02/reflexion04.htm. Acceso em 27 feb. 2018. 
ORGANIZACIÓN MUNDIAL DE LA SALUD. Carta de Ottawa para el Fomento de la Salud. Ginebra: OMS, 1986.

. Declaración de Helsinki sobre la salud en todas las politicas. Ginebra: OMS, 2013.

. Declaración de Yakarta sobre la Promoción de la Salud en el Siglo XXI. Ginebra:

OMS, 1997.

PAIVA, V. Analisando cenas e sexualidades: a promoção da saúde na perspectiva dos direitos humanos. In: CÁCARES, C. et al. (Eds.). Sexualidad, estigma y derechos humanos. Desafíos para el acceso a la salud en América Latina. Lima: FASPA/UPCH, 2006, p. 23-51.

POLLAND, B. Health promotion in Canada: perspectives and future prospects. Revista Brasileira em Promoção da Saúde, v. 20, n. 1, p. 3-11, 2007. Disponível em: <https://www. researchgate.net/publication/26456697_Health_Promotion_in_Canada_Perspectives_ Future_Prospects>. Acesso em: 27 feb. 2018.

SPINELLI, H. Las dimensiones del campo de la salud en Argentina. Salud Colectiva, Buenos Aires, v. 6, n. 3, p. 275-293, 2010. Disponível em: <http://www.scielo.org.ar/scielo. php?script=sci_arttext\&pid=S1851-82652010000300004>. Acesso em: 27 feb. 2018.

Wald, G. Arte y Salud: algunas reflexiones para profundizar las potencialidades de análisis del campo. Interface - Comunicação, saúde, educação, v. 19, n. 55, p. 1051-1062, Oct-dic 2015. Disponível em: <http://www.scielo.br/scielo.php?script=sci_arttext\&pid=S141432832015000401051\&lng=es\&nrm=iso >. Acesso em: 8 ene. 2019.

\section{Notas}

${ }^{1}$ Utilizamos el concepto de modelo en tanto instrumento metodológico para interpretar las prácticas. En tanto modelo supone un alto nivel de abstracción, y solo debe entenderse como referencia teórica de situaciones concretas, históricamente determinadas.

${ }^{2}$ Para ejemplos, ver: OMS (1986; 1997; 2013).

${ }^{3} \mathrm{G}$. Wald fue responsable de la redacción inicial de los ejes y principios del modelo, de la revisión de los pasos y de la elaboración del artículo. A. C. Camarotti fue responsable de la redacción inicial de los pasos del modelo, de la revisión de los ejes y principios y colaboró en la redacción del artículo. A. J. Capriati fue responsable de la redacción inicial de los pasos del modelo, de la revisión de los ejes y principios, y de la edición final de la versión a ser publicada. A. L. Kornblit fue responsable de la redacción inicial de los ejes y principios del modelo y de la revisión de los pasos. 


\section{Abstract}

\section{Community model for promoting, preventing, assisting and protecting children and adolescents from sexual abuse}

We present here a theoretical model for the construction of community responses to comprehensive adolescent health problems. It summarizes the work done by our team over the last 15 years, both in the field of research and in technical assistance to intervention programs in Argentina. The model is organized into 3 conceptual axes from which 10 principles and 6 steps emerge; it retrieves the debates of health promotion, collective health and critical pedagogy. Although it can be used to work on different problems of adolescent health, in this article it is applied to a specific topic of international relevance: sexual abuse against children and adolescents. This modeling implies a dialectic that, on the one hand, gives place so that the singular and contingent of each experience can appear. However, it recognizes that many problems are repeated in different communities, either close or geographically distant, and that the experience in dealing with them can contribute to build new action informed in practice. We propose here a work based on the inclusion of differences, where the myths, beliefs, prejudices and stereotypes that support the multiple discriminations that people can suffer are questioned.

> Keywords: model; health promotion; child sexual abuse; adolescents; community. 


\section{Resumo}

Modelo comunitário para promoção, prevenção, assistência e proteção ante situaçôes de abuso sexual de crianças e adolescentes Apresentamos um modelo teórico para a construção de respostas comunitárias a problemas de saúde integral do adolescente. $\mathrm{O}$ mesmo condensa o trabalho realizado por nossa equipe ao longo dos últimos 15 anos, tanto na área da pesquisa quanto na assistência técnica a programas de intervenção comunitária na Argentina. $\mathrm{O}$ modelo está organizado em três eixos conceptuais dos quais se desprendem dez princípios e seis passos, que recuperam os debates sobre a promoção da saúde, saúde coletiva e pedagogia crítica. Embora este possa ser utilizado para o trabalho com distintas problemáticas de saúde integral adolescente, é mostrado neste artigo aplicado a um tópico específico de relevância internacional: o abuso sexual contra crianças e adolescentes. O modelo supóe uma dialética que, por um lado, permite que o singular e contingente de cada experiência apareçam. Náo obstante, reconhece que muitos problemas se repitam em comunidades distintas, sejam perto ou geograficamente longe, e que a experiência na abordagem dos mesmos pode construir uma nova ação informada na prática. Proporemos aqui um trabalho baseado na inclusão das diferenças, em que se interroguem os mitos, crenças, preconceitos e estereótipos que sustentam as múltiplas discriminações que as pessoas podem sofrer.

Palavras-chave: modelo; promoção da saúde; abuso sexual infantil; adolescência; comunidade. 